\title{
Attitudes Toward COVID-19 Vaccines in Chinese Adolescents
}

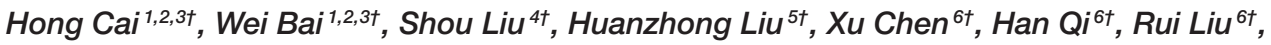
Teris Cheung ${ }^{7}$, Zhaohui $\mathrm{Su}^{8}$, Chee H. $\mathrm{Ng}^{9 *}$ and Yu-Tao Xiang ${ }^{1,2,3 *}$

${ }^{1}$ Unit of Psychiatry, Department of Public Health and Medicinal Administration \& Institute of Translational Medicine, Faculty of Health Sciences, University of Macau, Taipa, China, ${ }^{2}$ Centre for Cognitive and Brain Sciences, University of Macau, Taipa, China, ${ }^{3}$ Institute of Advanced Studies in Humanities and Social Sciences, University of Macau, Taipa, China, ${ }^{4}$ Department of Public Health, Medical College, Qinghai University, Xining, China, ${ }^{5}$ Department of Psychiatry, Chaohu Hospital of Anhui Medical University, Hefei, China, ${ }^{6}$ The National Clinical Research Center for Mental Disorders \& Beijing Key Laboratory of Mental Disorders, Beijing Anding Hospital \& the Advanced Innovation Center for Human Brain Protection, Capital Medical University, School of Mental Health, Beijing, China, ${ }^{7}$ School of Nursing, Hong Kong Polytechnic University, Kowloon, China, ${ }^{8}$ Center on Smart and Connected Health Technologies, Mays Cancer Center, School of Nursing, UT Health San Antonio, San Antonio, TX, United States, ${ }^{9}$ Department of Psychiatry, The Melbourne Clinic and St Vincent's Hospital, University of Melbourne, Richmond, VIC, Australia
\end{abstract}

\section{OPEN ACCESS}

Edited by:

Lin Wang,

University of Cambridge,

United Kingdom

Reviewed by:

Hideharu Hagiya,

Okayama University, Japan Juanjuan Zhang,

Fudan University, China

*Correspondence:

Yu-Tao Xiang

ytxiang@gmail.com

Chee H. Ng

cng@unimelb.edu.au

tThese authors have contributed equally to this work

Specialty section: This article was submitted to Infectious Diseases - Surveillance,

Prevention and Treatment, a section of the journal Frontiers in Medicine

Received: 05 April 2021 Accepted: 07 June 2021 Published: 07 July 2021

Citation:

Cai H, Bai W, Liu S, Liu H, Chen X,

Qi H, Liu R, Cheung T, Su Z, Ng CH and Xiang Y-T (2021) Attitudes Toward COVID-19 Vaccines in Chinese Adolescents. Front. Med. 8:691079. doi: 10.3389/fmed.2021.691079
Background: As COVID-19 vaccination programs are being implemented widely, it is important to examine the attitudes of adolescents toward the COVID-19 vaccine and its uptake. The aim of this study was to examine the acceptance of and attitudes toward COVID-19 vaccines, and their associated factors among adolescents in China.

Methods: This was a cross-sectional, observational study conducted between November 27, 2020 and March 12, 2021 using snowball sampling method. Basic sociodemographic characteristics, health-related information, severity of depressive and anxiety symptoms, and attitudes and behavior toward COVID-19 vaccines were assessed.

Results: Overall, 1,057 adolescents participated in this study, yielding a response rate of 89.3\%. There were 799 (75.59\%) [95\% Confidence Interval (Cl) 73.00-78.18\%] adolescents who would accept future COVID-19 vaccination. Binary logistic regression analysis revealed that adolescents who previously heard about COVID-19 vaccines $(P=0.001$, odds ratio $(O R)=1.90,95 \% C l: 1.32-2.74)$, who thought that COVID-19 vaccines could protect them from COVID-19 infection $(P=0.002$, OR $=2.93$, $95 \% \mathrm{Cl}: 1.49-5.70)$, and those who encouraged their family members and friends to get vaccinated $(P<0.001, \mathrm{OR}=12.19,95 \% \mathrm{Cl}$ : 6.78-21.92) and who believed that vaccines are safe $(P=0.012, \mathrm{OR}=3.94,95 \% \mathrm{Cl}: 1.36-11.44)$ were more likely to accept future COVID-19 vaccination. In addition, younger adolescents $(P=0.003, \mathrm{OR}$ $=0.93,95 \% \mathrm{Cl}$ : 0.89-0.98) were more likely to accept future COVID-19 vaccines than older adolescents.

Conclusions: In conclusion, Chinese adolescents appeared to have positive attitudes toward COVID-19 vaccines. It is important to increase public confidence and knowledge regarding the efficacy and safety of COVID-19 vaccines to maximize the success of vaccination programs.

Keywords: COVID-19 vaccines, adolescents, attitude toward vaccines, efficacy and safety of COVID-19 vaccines, acceptance of COVID-19 vaccines 


\section{INTRODUCTION}

Coronavirus disease 2019 (COVID-19) caused by Severe Acute Respiratory Syndrome Coronavirus 2 (SARS-CoV-2) has been declared a pandemic on March 11, 2020 by the World Health Organization (WHO) (1). As of March 15, 2021, there were over 120 million COVID-19 cases and more than 1 million deaths caused by COVID-19 reported (2). Although China has proactively controlled the rapid transmission of COVID19, sporadic cases of COVID-19 cases have persisted. Given the global impact of the COVID-19 pandemic on health and economy, effective infection control strategies to contain the virus transmission is the utmost priority among public health policymakers.

To date there are still no effective treatments for COVID$19(3,4)$. Vaccination is one of the most effective methods of controlling the COVID-19 pandemic by building herd immunity within the population. A multi-stage study comprising 9,542 residents in Wuhan, China found the proportion of participants who tested positive for COVID-19 pan-immunoglobulin was $6.92 \%$ (95\%CI: 6.42-7.43), which suggests that vaccination would be necessary to achieve herd immunity and avoid future COVID19 outbreaks (5). As of March 5, 2021, more than 200 vaccines were under development, of which more than 60 vaccines were in different stages of clinical trials, and at least seven vaccines have been rolled out (6). Many countries have started vaccination programs for certain subpopulations at high risk of infection (e.g., healthcare workers) in the first phase. In the next phase, other populations, such as healthy older adults, patients with chronic diseases, children and adolescents, will receive the COVID-19 vaccines (7). However, due to insufficient data on the efficacy and safety of vaccines in different subpopulations, the attitudes toward these COVID-19 vaccines have varied greatly (8).

According to a recent report examining the acceptability of COVID-19 vaccines among adults in the United States, 69\% of participants were willing to accept the COVID-19 vaccines (9). Another survey conducted in China found that $91.3 \%$ of participants would accept the COVID-19 vaccines (10). In addition, a large-scale study comprising 13,426 adults in 19 countries found that $69 \%$ were likely to accept the COVID19 vaccines (11). Commonly reported factors influencing vaccine acceptability and intention to be vaccinated included perceived benefit and efficacy of the COVID-19 vaccines $(9,10,12)$. However, to date no study has examined the acceptability of COVID-19 vaccines and its associated factors among adolescents. Students are one of the most susceptible subpopulations to the COVID-19 pandemic because of their immature immune system and their insufficient capacity for self-protection from communicable diseases. Therefore, it is important to understand the attitudes toward COVID-19 vaccines in this subpopulation to achieve the public health goals of vaccination programs.

In this study we aimed to evaluate the acceptance of and attitudes toward COVID-19 vaccines and their associated factors among Chinese adolescents.

\section{MATERIALS AND METHODS}

\section{Study Design}

This was a cross-sectional, observational study conducted between November 27, 2020 and March 12, 2021 using snowball sampling method through the collaborative research network of the National Clinical Research Center for Mental Disorders, China. To be eligible, participants should be secondary school students residing in China and able to understand the purpose and contents of the assessment. To avoid contagion during the COVID-19 pandemic, following previous studies $(13,14)$ data were collected online using the WeChat-based "Questionnaire Star" program. WeChat is a widely used smartphone-based social communication APP, with more than 1.2 billion active users in China. Following a previous study (15) using the WeChat-based "Questionnaire Star" program, all the questions in the assessments were set as "compulsorily answered" on an anonymous basis. Each assessment needed to be linked to a distinct IP address, and completed within a reasonable period based on a pilot study (the mean assessment time was $35 \mathrm{~min}$ in this study). All participants provided electronic written informed consent prior to participation in this study. Written consent from parents was sought for those participants aged under 18 years old. This study was approved by the Institutional Review Board (IRB) of Beijing Anding Hospital.

The sample size was calculated using the formula $\mathrm{N}=\mathrm{Z} \alpha^{2} \mathrm{P}$ $(1-\mathrm{P}) / \mathrm{d}^{2}$, in which $\alpha=0.05$ and $\mathrm{Z} \alpha=1.96$, and the estimated acceptable margin of error for proportion $d$ was 0.05 . The acceptance rate of COVID-19 vaccines was estimated to be $69 \%$ based on a previous study (11). Assuming that $10 \%$ of those invited would refuse participation in this study, at least 361 participants had to be included.

\section{Measures}

Basic sociodemographic characteristics and health related information were collected including age, gender, secondary school (junior/senior secondary school), residence (urban/rural), and perceived health status (bad/fair/good). Following a previous study on attitude about influenza vaccine (16), several standardized questions were added to measure attitudes and behaviors toward COVID-19 vaccines in this study, including: (1) "Do you worry about being infected with COVID-19?" (No/Fair/Very much); (2) "Have you heard of COVID-19 vaccines previously such as any information about COVID-19 vaccines, including both positive and negative news, vaccine development, safety and efficacy of vaccine via various channels (e.g., radio, television, telephone)?" (No/Yes); (3) "Do you think COVID-19 vaccines could protect you from COVID19?" (No/Yes/No idea); (4) "How safe do you think COVID-19 vaccines are?" (Not safe with obvious side effects/No idea/Safe with no or minimal side effects); (5) "Would you encourage your family and friends to get vaccinated with the COVID-19 vaccine?" (No/Yes/No idea). An extra question was asked to examine participants' behavior toward COVID-19 vaccines: "Do you intend to be vaccinated against COVID-19 in the future?" (No/Yes). 
TABLE 1 | Demographic characteristics of participants.

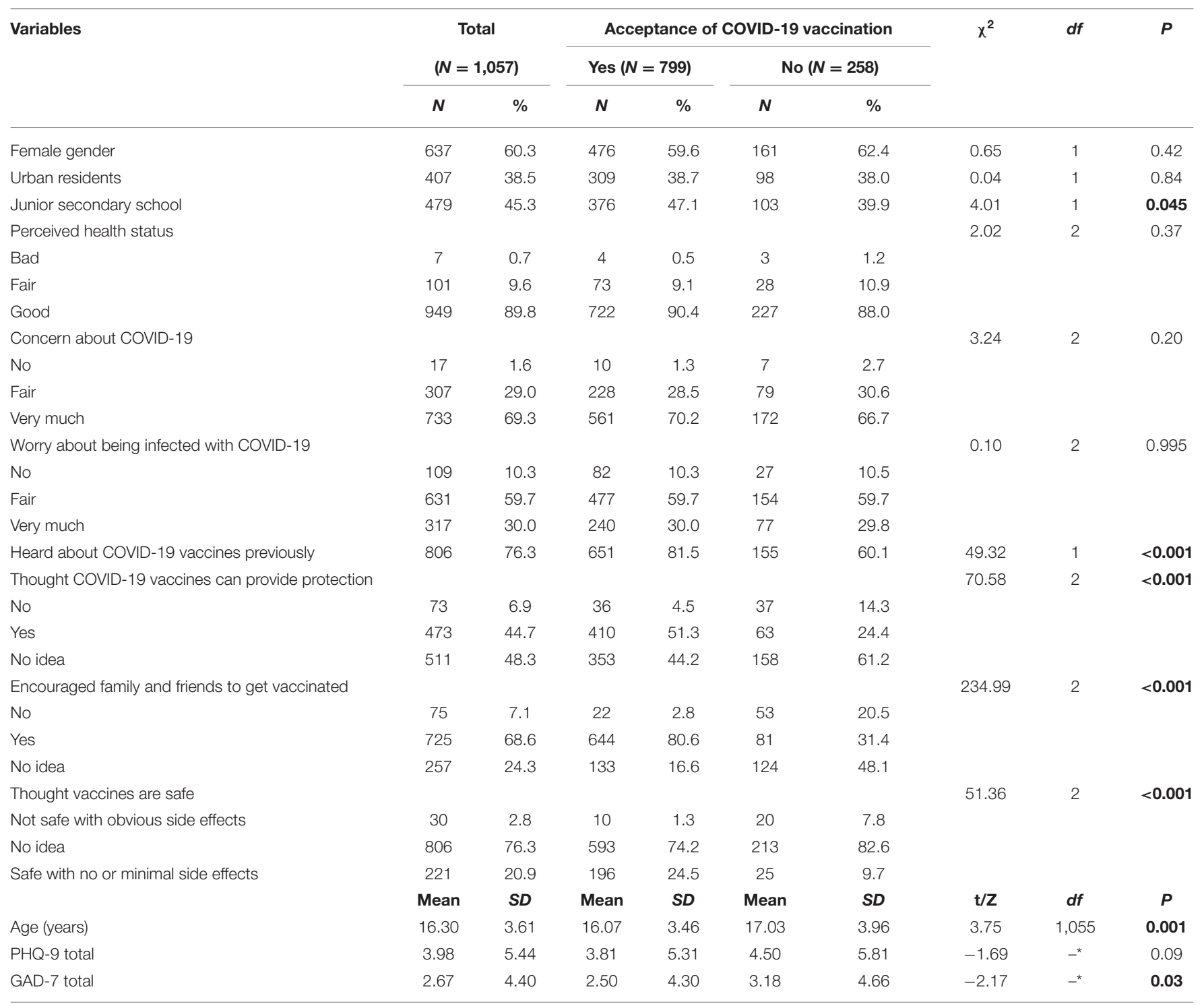

Bolded values, <0.05; M, mean; SD, standard deviation; COVID-19, Corona Virus Disease 2019; PHQ-9, 9-item Patient Health Questionnaire; GAD-7, 7-item Generalized Anxiety Disorder Scale.

"Mann-Whitney U-test.

Severity of the depressive symptoms was assessed using the Chinese version of the 9-item Patients Health Questionnaire (PHQ-9) $(17,18)$, with each item scoring from "0" (not at all) to "3" (nearly every day). The total scores ranged from 0 to 27 . A higher score indicates more severe depressive symptoms. Severity of anxiety symptoms was measured using the Chinese version of the 7-item Generalized Anxiety Disorder Scale (GAD-7) $(19,20)$, which comprises 7 items with each scoring from 0 (not at all) to 3 (nearly every day) with a total score ranging from 0 to 21 . A higher score indicates more severe anxiety symptoms.

\section{Statistical Analysis}

Data analyses were performed using SPSS version 25.0 (SPSS Inc., Chicago, Illinois, USA). All continuous variables were checked for normal distributions using P-P plots. Chi-square tests, independent samples $t$-tests, and Mann-Whitney $U$-tests were used to compare socio-demographic and COVID-19 vaccine related variables between adolescents who would accept future COVID-19 vaccination and those who would not accept. In order to examine the independent correlates of acceptance of future COVID-19 vaccination, the variables that significantly differed in univariate analyses were entered as independent variables in binary logistic analysis with the "enter" method. Significant statistical difference was set at $P<0.05$ (two-tailed).

\section{RESULTS}

Overall, 1,183 adolescents were invited to participate in the survey, and 1,057 completed the survey, yielding a response rate of $89.3 \%$. There were 799 (75.59\%, 95\%CI: $73.00-78.18 \%)$ 


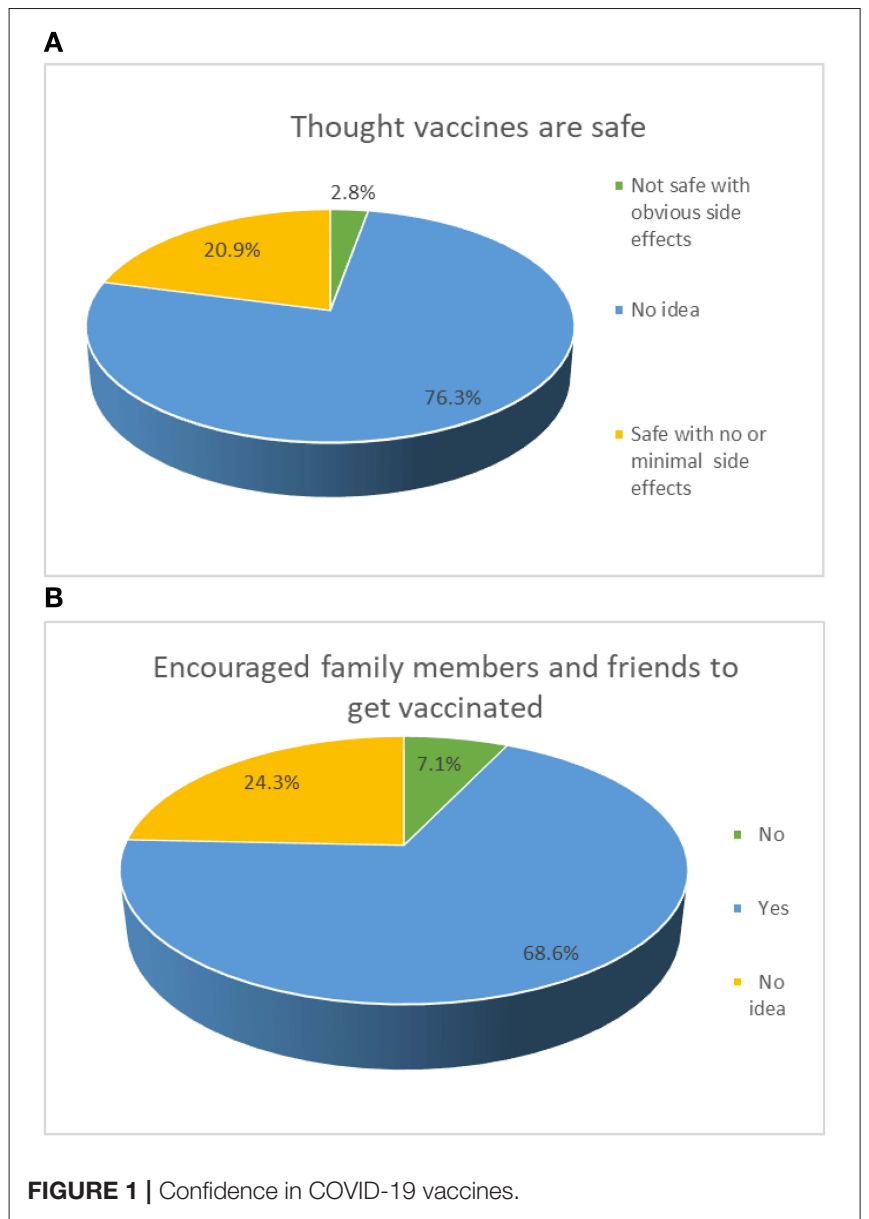

adolescents who would accept future COVID-19 vaccination. The mean age was 16.30 [standard deviation (SD): 3.61 ] years (range: $12-20$ years) and $637(60.3 \%)$ participants were female (Table 1). Of the participants, $76.3 \%$ (95\%CI: 73.69-78.82\%) believed that COVID-19 vaccines are safe with no or mild side effects, while 68.6\% (95\%CI: 65.79-71.39\%) would encourage family and friends to get vaccinated with the COVID-19 vaccines (Figure 1).

Table 1 shows the demographic characteristics and health related information of the participants and the comparisons between adolescents who would accept future COVID-19 vaccination and those who would not accept. There were significant differences between groups in terms of educational level, age, and anxiety symptoms, as well as the responses to the four questions about attitudes toward COVID-19 vaccines. No difference in depression was found between adolescents who accepted and those who did not accept future COVID-19 vaccination $(p=0.09)$.

Binary logistic regression analysis revealed that adolescents who heard about COVID-19 vaccines previously $(P=0.001$, OR $=1.90$, 95\%CI:1.32-2.74), who thought COVID-19 vaccines can provide protection $(P=0.002$, OR $=2.93,95 \% \mathrm{CI}$ : $1.49-5.70)$, who encouraged family members and friends to get vaccinated
TABLE 2 | Independent correlates of future COVID-19 vaccination acceptance by multiple logistic regression analysis.

\begin{tabular}{|c|c|c|c|}
\hline \multirow[t]{2}{*}{ Variables } & \multicolumn{3}{|c|}{ Multiple logistic regression analysis } \\
\hline & $\boldsymbol{P}$-values & OR & $95 \% \mathrm{Cl}$ \\
\hline Junior secondary schools & 0.88 & 1.03 & $0.72-1.47$ \\
\hline Heard about COVID-19 vaccines previously & 0.001 & 1.90 & $1.32-2.74$ \\
\hline \multicolumn{4}{|c|}{ Thought COVID-19 vaccines can provide protection } \\
\hline No & - & 1.0 & - \\
\hline Yes & 0.002 & 2.93 & $1.49-5.70$ \\
\hline No idea & 0.033 & 2.04 & $1.06-3.93$ \\
\hline \multicolumn{4}{|c|}{ Encouraged family members and friends to get vaccinated } \\
\hline No & - & 1.0 & - \\
\hline Yes & $<0.001$ & 12.19 & $6.78-21.92$ \\
\hline No idea & 0.026 & 2.00 & $1.09-3.67$ \\
\hline \multicolumn{4}{|l|}{ Thought vaccines are safe } \\
\hline Not safe with obvious side effects & - & 1.0 & - \\
\hline No idea & 0.016 & 3.30 & $1.25-8.74$ \\
\hline Safe with no or minimal side effects & 0.012 & 3.94 & $1.36-11.44$ \\
\hline Age (years) & 0.003 & 0.93 & $0.89-0.98$ \\
\hline GAD-7 total & 0.27 & 0.98 & $0.95-1.02$ \\
\hline
\end{tabular}

Bolded values, <0.05; Cl, confidential interval; OR, odds ratio; COVID-19, Corona Virus Disease 2019; GAD-7, 7-item Generalized Anxiety Disorder Scale.

$(P<0.001$, OR $=12.19,95 \%$ CI: 6.78-21.92 $)$ and who thought vaccines are safe $(P=0.012, \mathrm{OR}=3.94,95 \% \mathrm{CI}$ : $1.36-11.44)$ were more likely to accept future COVID-19 vaccination (Table 2). In addition, younger adolescents $(P=0.003$, OR $=0.93$, 95\%CI: 0.89-0.98) were more likely to accept future COVID19 vaccination than older adolescents. No association between anxiety and COVID-19 vaccine acceptance $(p=0.27)$ was found (Table 2).

\section{DISCUSSION}

To the best of our knowledge, this was the first study that examined the attitudes of adolescents toward COVID-19 vaccines and their associated factors. Our findings were similar to other international findings that around $69-80 \%$ of adults would accept COVID-19 vaccination in countries such as England, Denmark, the US, Australia and France (9, 11, 21-24). In addition, no associations of COVID-19 vaccine acceptance with anxiety or depression were observed in this survey. However, our results were lower than the previous figures found among Chinese adults who had a COVID-19 vaccination acceptance rate of $91.3 \%$ (10). The main reasons for the lower vaccine acceptance rate in adolescents may include uncertainty about vaccine safety and efficacy, and inadequate knowledge about the potential benefits of vaccination among children and adolescents (21). In addition, insufficient clinical trial data, lack of knowledge about the vaccine and the COVID-19 may increase misconceptions about COVID-19 vaccines (25). Further, the possibility of pain and discomfort associated with vaccination could also lead to a lower acceptance rate of the COVID-19 vaccines in adolescents. 
In this study, we found that younger adolescents were more likely to accept COVID-19 vaccination than older adolescents, possibly because younger adolescents are more compliant with positive directions and had limited access to online negative information or misinformation about vaccines than older adolescents.

Adolescents who heard about COVID-19 vaccines previously were more likely to accept future COVID-19 vaccination, which reflects a better recognition of the efficacy of vaccines in controlling the pandemic. In response to the profound impact of the pandemic, China has taken rigorous public health interventions to control the spread of COVID-19 since the outbreak of the disease $(26,27)$. Chinese residents often hold strong beliefs about the efficacy of COVID-19 vaccination and such a positive attitude may explain why adolescents who heard about COVID-19 vaccines previously were more likely to accept COVID-19 vaccination. According to the health belief model, the perceived benefit from vaccination is likely to outweigh the risks (28-30). As of March 15, 2021, nearly 65 million people in China have been vaccinated against COVID-19 (6, 8), with no severe adverse reactions reported so far. Further, in China tertiary general hospitals are located close to the vaccination sites which could provide timely and professional interventions to identify and respond to any adverse reactions from the COVID-19 vaccines. All these factors could increase public confidence of the COVID-19 vaccination programs among residents including adolescents.

Public concern about vaccine safety has frequently been reported as a major obstacle to the rollout of vaccination programs, especially for newly introduced vaccines in clinical practice $(28,31,32,36)$. For example, an online survey at the start of the vaccination program in China revealed that only $54.8 \%$ of the participants had ("probably yes") intent to be vaccinated against COVID-19 (12). Positively, China has since established a vaccine regulatory system, with relevant vaccine regulations and a quality control system. Therefore, these safeguards around the development and implementation of the COVID-19 vaccination programs have increased the overall vaccine acceptance in the community at large.

Adolescents who encouraged family members and friends to get vaccinated were more likely to accept future COVID19 vaccination. This may be largely driven by their concern to protect their family members and friends from contracting COVID-19. If participants believe that their family members and friends are in the high-risk groups, they would encourage them to have the COVID-19 vaccination (33). Studies have shown that parents' values and attitudes can be influenced by their children $(34,35)$. For instance, in a study conducted in eastern Zambia, school-age children and adolescents are change agents who had a positive impact on their families' or friends' health behaviors such as the practice of water, sanitation, and personal hygiene (34).

The strengths of this study included the large sample size and the focus on the adolescent population covering many provinces in China. However, there are several limitations that should be addressed. First, due to the cross-sectional study design, the casual relationship between attitudes toward COVID-19 vaccines and other variables could not be established. Second, although the attitudes toward COVID-19 vaccines in adolescents may largely depend on their parents or guardians, we did not measure the attitudes of the parents or legal guardians of the participants. Hence, the study could not reflect the potential coverage of vaccines in adolescents in the future, similar to previous studies (21). Third, due to logistical reasons, participants were recruited by a snowball, rather than random sampling method, which may limit the generalizability of the findings. Fourth, there were several minor outbreaks caused by imported cases from overseas in January 2021 in Hebei, Heilongjiang, and Jilin provinces, which may have influenced the adolescents' attitudes toward COVID-19 vaccines to an uncertain extent. Lastly, the standardized questions on attitudes and behaviors toward COVID-19 vaccines used in this survey were based on a previous study on attitude about influenza vaccine (16). Hence, certain additional information, such as having no idea about the attitude toward COVID-19 vaccines, could not be included in the study.

In conclusion, Chinese adolescents appeared to have a relatively high rate of acceptance of COVID-19 vaccination. It is important to increase the public confidence and knowledge regarding the efficacy and safety of COVID-19 vaccines to maximize the success of vaccination programs.

\section{DATA AVAILABILITY STATEMENT}

The Institutional Review Board (IRB) of Beijing Anding Hospital that approved the study prohibits the authors from making the research data set publicly available. Readers and all interested researchers may contact Dr. Yu-Tao Xiang (Email address: xyutly@gmail.com) for details. Dr. Xiang could apply to the Institutional Review Board (IRB) of Beijing Anding Hospital for the release of the data.

\section{ETHICS STATEMENT}

The studies involving human participants were reviewed and approved by the Institutional Review Board (IRB) of Beijing Anding Hospital. Written informed consent to participate in this study was provided by the participants' legal guardian/next of kin.

\section{AUTHOR CONTRIBUTIONS}

Y-TX and CN: study design. HC, WB, SL, HL, XC, HQ, and RL: collection, analyses, and interpretation of data. $\mathrm{HC}, \mathrm{CN}$, and Y-TX: drafting of the manuscript. TC and ZS: critical revision of the manuscript. All the authors approval of the final version for publication.

\section{FUNDING}

This study was supported by the National Science and Technology Major Project for investigational new drug (2018ZX09201-014), the Beijing Municipal Science \& Technology Commission (No. Z181100001518005), and the University of Macau (MYRG2019-00066-FHS). 


\section{REFERENCES}

1. World Health Organization. Coronavirus disease (COVID-19) Pandemic. World Health Organization (2020). Available online at: https://www.who.int/ emergencies/diseases/novel-coronavirus-2019 (accessed March 21, 2021).

2. World Health Organization. COVID-19 Reports (2021). Available online at: https://www.who.int/emergencies/diseases/novel-coronavirus-2019/ situation-reports (accessed March 15, 2021).

3. Huang CW, Li Y, Ren X, Zhao L, Hu J, Zhang Y, et al. Clinical features of patients infected with 2019 novel coronavirus in Wuhan, China. Lancet. (2020) 395:497-506. doi: 10.1016/S0140-6736(20)30183-5

4. World Health Organization. Technical Guidelines on Coronavirus Disease 2019 (COVID-19) (2020). Available online at: https://www.who.int/zh/ emergencies/diseases/novel-coronavirus-2019/technical-guidance (accessed March 21, 2021).

5. Richard Stugnell NW. Seroprevalence and Humoral Immune Durability of Anti-SARS-CoV-2 Antibodies in Wuhan, China: A Longitudinal, Population-Level, Cross-Sectional Study. Lancet. (2021) 397:1075-84. doi: 10.1016/S0140-6736(21)00238-5

6. World Health Organization. COVID-19 Vaccines (2021). Available online at: https://www.who.int/zh/emergencies/diseases/novel-coronavirus-2019/ covid-19-vaccines (accessed March 21, 2021).

7. XinJing Net. Can the Second-Dose Vaccine Be Vaccinated from Different Manufacturer? The Latest 10 Questions About New COVID-19 Vaccination (2021). Available online at: https://www.bjnews.com.cn/detail/ 161692031715064.html (accessed March 28, 2021).

8. XinHua Net. My Country's New Coronavirus Vaccine Has Been Vaccinated for Nearly 65 Million People (2021). Available online at: http://www.xinhuanet. com/2021-03/16/c_1127214918.htm (accessed March 16, 2021).

9. Reiter PL, Pennell ML, Katz ML. Acceptability of a COVID-19 vaccine among adults in the United States: how many people would get vaccinated? Vaccine. (2020) 38:6500-7. doi: 10.1016/j.vaccine.2020.08.043

10. Wang J, Jing R, Lai X, Zhang H, Lyu Y, Knoll MD, et al. Acceptance of COVID19 Vaccination during the COVID-19 pandemic in China. Vaccines. (2020) 8:482. doi: $10.3390 /$ vaccines 8030482

11. Lazarus JVR, Palayew SC, Gostin A, Larson LO, Rabin HJ, Kimball K, et al. A global survey of potential acceptance of a COVID-19 vaccine. Nat Med. (2021) 27:225-8. doi: 10.1038/s41591-020-1124-9

12. Lin $\mathrm{Y}, \mathrm{Hu} Z$, Zhao Q, Alias H, Danaee M, Wong LP. Understanding COVID19 vaccine demand and hesitancy: a nationwide online survey in China. PLoS Negl Trop Dis. (2020) 14:e0008961. doi: 10.1371/journal.pntd.0008961

13. Luo H, Lie Y, Prinzen FW. Surveillance of COVID-19 in the general population using an online questionnaire: report from 18,161 respondents in China. JMIR Public Health Surv. (2020) 6:e18576. doi: 10.2196/18576

14. Zhou J, Liu L, Xue $\mathrm{P}$, Yang $\mathrm{X}$, Tang $\mathrm{X}$. Mental health response to the COVID-19 outbreak in China. Am J Psychiatry. (2020) 177:5745. doi: 10.1176/appi.ajp.2020.20030304

15. Chen R, Zhu X, Wright L, Drescher J, Gao Y, Wu L, et al. Suicidal ideation and attempted suicide amongst Chinese transgender persons: national population study. J Affect Disord. (2019) 245:1126-34. doi: 10.1016/j.jad.2018.12.011

16. Ma Y, Li T, Chen W, Chen J, Li M, Yang Z. Knowledge, Attitudes and Practices (KAP) toward seasonal influenza vaccine among young workers in South China. Hum Vaccin Immunother. (2018) 14:128393. doi: 10.1080/21645515.2017.1423157

17. Kroenke K, Spitzer RL, Williams JB. The PHQ-9: validity of a brief depression severity measure. J Gen Intern Med. (2001) 16:606-13. doi: 10.1046/j.1525-1497.2001.016009606.x

18. Zhang YL, Liang W, Chen ZM, Zhang HM, Zhang JH, Weng XQ, et al. Validity and reliability of patient health questionnaire- 9 and patient health questionnaire-2 to screen for depression among college students in China. Asia Pac Psychiatry. (2013) 5:268-75. doi: 10.1111/appy. 12103

19. He XY, Li CB, Qian J, Cui HS, Wu WY. Reliability and validity of a generalized anxiety scale in general hospital outpatients (in Chinese). Shanghai Archiv Psychiatry. (2010) 22:200-3. doi: 10.3969/j.issn.1002-0829.2010.04.002

20. Spitzer RL, Kroenke K, Williams JB, Lowe B. A brief measure for assessing generalized anxiety disorder: the GAD-7. Arch Intern Med. (2006) 166:10927. doi: 10.1001/archinte.166.10.1092
21. Bell S, Clarke R, Mounier-Jack S, Walker JL, Paterson P. Parents' and guardians' views on the acceptability of a future COVID19 vaccine: a multi-methods study in England. Vaccine. (2020) 38:7789-98. doi: 10.1016/j.vaccine.2020.10.027

22. Dodd RH, Cvejic E, Bonner C, Pickles K, McCaffery KJ, Sydney Health Literacy Lab COVID-19 group. (2021). Willingness to vaccinate against COVID-19 in Australia. Lancet Infect Dis 21, 318-9. doi: 10.1016/S1473-3099(20)30559-4

23. Neumann-Bohme SV, Sabat NE, Barros I, Brouwer PP, van Exel W, Schreyogg J, et al. Once we have it, will we use it? A European survey on willingness to be vaccinated against COVID-19. Eur J Health Econ. (2020) 21:97782. doi: 10.1007/s10198-020-01208-6

24. The COCONEL Group. A future vaccination campaign against COVID19 at risk of vaccine hesitancy and politicisation. Lancet Infect Dis. (2020) 20:769-70. doi: 10.1016/S1473-3099(20)30426-6

25. Maisonneuve AR, Witteman HO, Brehaut J, Dube E, Wilson K. Educating children and adolescents about vaccines: a review of current literature. Expert Rev Vaccines. (2018) 17:311-21. doi: 10.1080/14760584.2018.1456921

26. Kraemer MUGY, Gutierrez CH, Wu B, Klein CH, Pigott B, Open DM, et al. The effect of human mobility and control measures on the COVID-19 epidemic in China. Science. (2020) 368:493-7. doi: 10.1126/science.abb4218

27. Tian HL, Li Y, Wu Y, Chen CH, Kraemer B, Li MUG, et al. An investigation of transmission control measures during the first 50 days of the COVID-19 epidemic in China. Science. (2020) 368:638-42. doi: 10.1126/science.abb6105

28. Lau JT, Yeung NC, Choi KC, Cheng MY, Tsui HY, Griffiths S. Factors in association with acceptability of $\mathrm{A} / \mathrm{H} 1 \mathrm{~N} 1$ vaccination during the influenza $\mathrm{A} / \mathrm{H} 1 \mathrm{~N} 1$ pandemic phase in the Hong Kong general population. Vaccine. (2010) 28:4632-7. doi: 10.1016/j.vaccine.2010.04.076

29. Raude J, Caille-Brillet AL, Setbon M. The 2009 pandemic H1N1 influenza vaccination in France: who accepted to receive the vaccine and why? PLoS Curr. (2010) 2:RRN1188. doi: 10.1371/currents.RRN1188

30. Slater PE, Weidberg-Shmerler B, Morag A. Underutilization of influenza vaccine in Jerusalem. Public Health Rev. (1995) 23:357-66.

31. Nguyen T, Henningsen KH, Brehaut JC, Hoe E, Wilson K. Acceptance of a pandemic influenza vaccine: a systematic review of surveys of the general public. Infect Drug Resist. (2011) 4:197-207. doi: 10.2147/IDR.S23174

32. Yaqub O, Castle-Clarke S, Sevdalis N, Chataway J. Attitudes to vaccination: a critical review. Soc Sci Med. (2014) 112:111. doi: 10.1016/j.socscimed.2014.04.018

33. Williams L, Gallant AJ, Rasmussen S, Brown Nicholls LA, Cogan N, Deakin $\mathrm{K}$, et al. Towards intervention development to increase the uptake of COVID19 vaccination among those at high risk: outlining evidence-based and theoretically informed future intervention content. Br J Health Psychol. (2020) 25:1039-54. doi: 10.1111/bjhp.12468

34. Bresee S, Caruso BA, Sales J, Lupele J, Freeman MC. 'A child is also a teacher': exploring the potential for children as change agents in the context of a schoolbased WASH intervention in rural Eastern Zambia. Health Educ Res. (2016) 31:521-34. doi: 10.1093/her/cyw022

35. Vaughan C, Gack J, Solorazano H, Ray R. The effect of environmental education on schoolchildren, their parents, and community members: a study of intergenerational and intercommunity learning. J Environ Educ. (2003) 34:12-21. doi: 10.1080/00958960309603489

36. Schwarzinger M, Flicoteaux R, Cortarenoda S, Obadia Y, Moatti JP. Low acceptability of $\mathrm{A} / \mathrm{H} 1 \mathrm{~N} 1$ pandemic vaccination in French adult population: did public health policy fuel public dissonance? PLoS ONE. (2010) 5:e10199. doi: 10.1371/journal.pone.0010199

Conflict of Interest: The authors declare that the research was conducted in the absence of any commercial or financial relationships that could be construed as a potential conflict of interest.

Copyright (c) 2021 Cai, Bai, Liu, Liu, Chen, Qi, Liu, Cheung, Su, Ng and Xiang. This is an open-access article distributed under the terms of the Creative Commons Attribution License (CC BY). The use, distribution or reproduction in other forums is permitted, provided the original author(s) and the copyright owner(s) are credited and that the original publication in this journal is cited, in accordance with accepted academic practice. No use, distribution or reproduction is permitted which does not comply with these terms. 